Effect of the directional blood flow on thermal dose distribution during thermal therapy: an application of a Green's function based on the porous model

This article has been downloaded from IOPscience. Please scroll down to see the full text article.

2003 Phys. Med. Biol. 481577

(http://iopscience.iop.org/0031-9155/48/11/307)

The Table of Contents and more related content is available

Download details:

IP Address: 140.112.113.225

The article was downloaded on 06/01/2009 at 06:03

Please note that terms and conditions apply. 


\title{
Effect of the directional blood flow on thermal dose distribution during thermal therapy: an application of a Green's function based on the porous model
}

\author{
Hong-Sen Kou ${ }^{1}$, Tzu-Ching Shih ${ }^{1}$ and Win-Li Lin ${ }^{2}$ \\ ${ }^{1}$ Department of Mechanical Engineering, Tatung University, Taipei, Taiwan \\ ${ }^{2}$ Institute of Biomedical Engineering, National Taiwan University, Taipei, Taiwan \\ E-mail: hskou@ttu.edu.tw
}

Received 13 January 2003

Published 20 May 2003

Online at stacks.iop.org/PMB/48/1577

\begin{abstract}
This study presents the effects of directional blood flow and heating schemes on the distributions of temperature and thermal dose during thermal therapy. In this study, a transient bioheat transfer equation based on the porous medium property is proposed to encompass the directional effect of blood flow. A Green's function is used to obtain the temperature distribution for this modified bioheat transfer equation, and the thermal dose equivalence is used to evaluate the heating results for a set of given parameters. A $10 \times 10 \times 10 \mathrm{~mm}^{3}$ tumour tissue is heated by different heating schemes to investigate the thermal dose variation with the clinical therapeutic arrangement. For a rapid heating scheme, the domain of thermal lesion can effectively cover the desired therapeutic region. However, this domain of thermal lesion may extend to the downstream normal tissue if the porosity is high and the averaged blood velocity has a larger value.
\end{abstract}

\section{Introduction}

Thermal therapy utilizes the cytotoxic effect of high temperature to destroy pathological tissues. Conventional hyperthermia, with target temperatures of $41-45^{\circ} \mathrm{C}$ and duration ranging from 30 min to several hours, in combination with radiation, has demonstrated increasing effectiveness in thermal treatments (Overgaard et al 1996). In contrast, the use of temperatures above $55^{\circ} \mathrm{C}$ can directly destroy tissues through thermal coagulation (Beacco et al 1994). This higher temperature can deliver enough energy to denature proteins and result in complete cell death in the heated region within seconds (Thomsen 1991). Moreover, thermal lesion dimensions produced by high temperature thermal therapy correlate well with prediction 
based on the calculation of the transient temperature distribution (Damianou et al 1995, Kolios et al 1996). Therefore, it is important to understand the transient temperature behaviour in heated tissues during the heating process.

In general, the temperature distributions inside the heated tissues, controlled by heating modalities, are obtained by solving the bioheat transfer equation (BHTE), as proposed by Pennes in 1948 under appropriate initial and boundary conditions. A more complex framework for solving the BHTE by using a time-dependent Green's function and Fourier transform techniques was established by Gao et al (1995). Since the Green's function obtained from the partial differential equation is independent of the source term, the same Green's function is used to calculate temperature distributions with various heating patterns. However, the major criticism of the conventional Pennes' model focused on the assumption that the heat transfer by blood flow occurs in a non-directional, heat sink- or source-like term (Wulff 1974). Weinbaum et al (1984a, 1984b) and Chen and Holmes (1980) attempted to formulate a more realistic bioheat equation, which would account for the thermally convective contribution of the vasculature in addition to the heat sink- or source-like term originally proposed by Pennes (1948).

Blood flow plays an important role in determining the effectiveness of thermal therapy, both for hyperthermia alone, and in combination with either radiation or chemotherapy. In general, the effect of blood flow on temperature distribution in tissue is considered as cooling due to both thermally significant large vessels and smaller microvasculature during thermal treatments (Baish 1994). Furthermore, Kolios et al (1995) examined the effect of the thermally significant large blood vessels on the thermal distributions during thermal therapy. The dissipation of heat from heated tissues is carried out by convection through blood flow and also by conduction or diffusion processes. Kolios et al (1999) found that large vessels still have pronounced effect on the tissue temperature distribution even when the treatment time is within 3-20 s.

Detailed descriptions of the development and morphological characteristics of tumour vasculature and tumour blood flow have been extensively investigated by Folkman (1986) and Shubik (1982). The growth of a larger solid tumour requires nutrition from adequate vascular distribution and blood flow. By morphometric analysis of the microvasculature of tumours (C3H/Bi mammary carcinomas), Hilmas and Gillette (1974) found that the changes in vascular parameters during tumour growth ranged from $4 \mathrm{~mm}$ average diameter (approximately $35 \mathrm{~mm}^{3}$ ) to $14 \mathrm{~mm}$ average diameter (approximately $1500 \mathrm{~mm}^{3}$ ). Furthermore, Hirst (1989) found that the blood supply to tumours can be classified into three broad categories with important implications for the effects of vasoactive agents. He modelled the vascular beds assuming that the relationship between the tumour vascular bed and the normal tissue vascular bed is 'in parallel', 'in series' or 'combined'. Hirst et al (1992) studied the tumour vascular physiology to gain a better understanding of the rules that determine how blood will flow through tumours of different types, sizes and growth sites in response to changes in blood pressure, vascular tone and blood viscosity. Lacking smooth muscle layers and innervations, the thin capillary-like tumour vessels are unable to autoregulate (Molls and Vaupel 1998). According to Hirst's tumour vascular modelling, a heating target volume containing the capillary-like tumour vascular bed with directional blood flow is considered as shown in figure 1. In some studies (Wan et al 1996, Fan and Hynynen 1996, Daum and Hynynen 1999, Wu and Sherar 2002) on ultrasound surgery, they could successfully treat a $10 \times 10 \times 10 \mathrm{~mm}^{3}$ tumour tissue. Baish et al (1997) used an isotropic porous medium concept to describe the transmural coupling of fluid flow in a microcirculatory network and interstitium in tumours. They assumed that the vascular bed of tumour tissue can be modelled as a network of vessels connecting the arterial (upstream) and venous (downstream) ends. In this study we chose a $10 \times 10 \times 10 \mathrm{~mm}^{3}$ target 


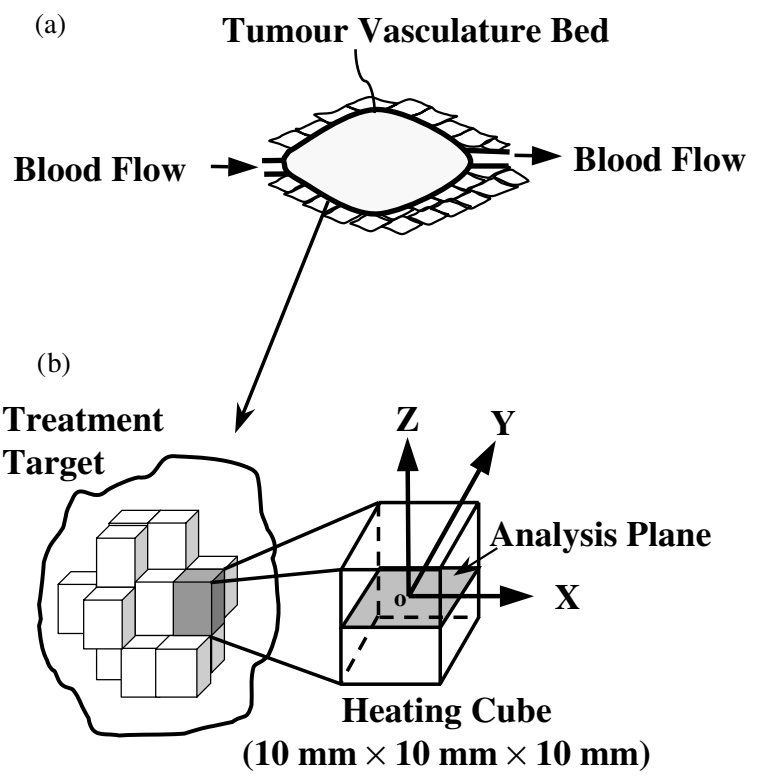

Figure 1. Schematic diagram of the geometry studied for the distributions of the temperature and thermal dose equivalence $\left(\mathrm{EM}_{43}\right)$ in the tissue. (a) Geometric relationship between the tumour vascular bed and the directional blood flow (modelled after Hirst (1989)), presenting a schematic diagram of the directional characteristics of the vascular bed. (b) The large treatment tumour (volume enclosed by the coarse line) with one-way directional blood flow was divided into several small heating cubes. A simulated heated cube was assumed to be of dimensions $10 \mathrm{~mm} \times 10 \mathrm{~mm} \times$ $10 \mathrm{~mm}$. The initial temperatures of the selected heating cube and the surrounding tissue were set to $37^{\circ} \mathrm{C}$.

tissue volume with directional tumour blood flow to analyse the thermal distributions during thermal therapies.

Since there is little information concerning the effects of blood flow direction on the region of the thermal lesion, especially for transient and three-dimensional calculations, the purpose of this study is to investigate the effect of blood flow direction and porosity on thermal distributions within the heated tumour volume, and to use the Green's function to solve the analytical solution of the modified bioheat transfer equation based on the porous medium that encompasses the directional effect of the blood flow.

\section{Methods}

\subsection{Derivation of heat transfer in the blood-perfused tumour tissue}

Heat transfer in living tissue can be modelled by solving a conjugate problem with the porous medium concept. This model contains a solid sub-domain for the tissue and a liquid subdomain for the blood. Herein the vascular volume in solid tissues is fully occupied with blood flow. That is, we assume that the vascular volume is fully equal to the blood volume. Heat transfer in blood-perfused tumour tissue volume can be modelled by solving a conjugate problem containing a solid sub-volume (the tissue domain) and a liquid sub-volume (the blood domain). The energy transport equations of the tissue and the blood flow domains are used 
Table 1. Parameters used for the numerical simulations.

\begin{tabular}{llll}
\hline Symbol & Definition & Value & Units \\
\hline$\rho_{\mathrm{t}}, \rho_{\mathrm{b}}$ & Density of tissue and blood & 1050 & $\mathrm{~kg} \mathrm{~m}^{-3}$ \\
$c_{\mathrm{t}}, c_{\mathrm{b}}$ & Specific heat of tissue and blood & $3770^{\mathrm{a}}$ & $\mathrm{J} \mathrm{kg}^{-1}{ }^{\circ} \mathrm{C}^{-1}$ \\
$t$ & Time & Variable & $\mathrm{s}$ \\
$T$ & Temperature & Calculated & ${ }^{\circ} \mathrm{C}$ \\
$k_{\mathrm{t}}, k_{\mathrm{b}}$ & Thermal conductivity of tissue and blood & $0.5^{\mathrm{b}}$ & $\mathrm{W} \mathrm{m}^{-1}{ }^{\circ} \mathrm{C}^{-1}$ \\
$u$ & Average blood velocity & $0.3-2^{\mathrm{c}}$ & $\mathrm{mm} \mathrm{s}^{-1}$ \\
$T_{\mathrm{a}}$ & Arterial temperature & 37 & ${ }^{\circ} \mathrm{C}$ \\
$q$ & Heating power & Variable & $\mathrm{W} \mathrm{cm}$ \\
\hline a Damianou and Hynynen (1993). & & \\
$\mathrm{b}$ Fan and Hynynen (1996). & & \\
${ }^{\mathrm{c}}$ Guyton (1986), Nilsson (1996), Keller and Seiler (1971), Crezee and Lagendijk (1992) and \\
Hobbie (1997).
\end{tabular}

here as shown in equations (1) and (2), respectively,

$\rho_{\mathrm{t}} c_{\mathrm{t}} \frac{\partial T_{\mathrm{t}}}{\partial t}=k_{\mathrm{t}}\left(\frac{\partial^{2} T_{\mathrm{t}}}{\partial x^{2}}+\frac{\partial^{2} T_{\mathrm{t}}}{\partial y^{2}}+\frac{\partial^{2} T_{\mathrm{t}}}{\partial z^{2}}\right)-W_{\mathrm{b}} c_{\mathrm{b}}\left(T_{\mathrm{t}}-T_{\mathrm{b}}\right)+q_{\mathrm{t}}(x, y, z, t)$
$\rho_{\mathrm{b}} c_{\mathrm{b}}\left(\frac{\partial T_{\mathrm{b}}}{\partial t}+u \frac{\partial T_{\mathrm{b}}}{\partial x}+v \frac{\partial T_{\mathrm{b}}}{\partial y}+w \frac{\partial T_{\mathrm{b}}}{\partial z}\right)=k_{\mathrm{b}}\left(\frac{\partial^{2} T_{\mathrm{b}}}{\partial x^{2}}+\frac{\partial^{2} T_{\mathrm{b}}}{\partial y^{2}}+\frac{\partial^{2} T_{\mathrm{b}}}{\partial z^{2}}\right)+q_{\mathrm{b}}(x, y, z, t)$

where $\rho$ is the density, $c$ is the specific heat, $k$ is the thermal conductivity, $T$ is the temperature, $q(x, y, z, t)$ is the absorbed power density with the subscripts $\mathrm{t}$ and $\mathrm{b}$ referring to tissue and blood domain, respectively, and $W_{\mathrm{b}}$ is the blood perfusion rate. All units and values of the above parameters are given in table 1. Similar equations had been found in Kolios et al (1995) that were valid for cylindrical coordinates and axial flow. In the report of Hilmas and Gillette (1974), it is observed that the tumour vessel diameter in a $1000 \mathrm{~mm}^{3}$ tumour volume is less than $30 \mu \mathrm{m}$. This means that thermal equilibrium occurs in this region and thereafter we also choose a $10 \times 10 \times 10 \mathrm{~mm}^{3}$ heated cube as the target volume. In addition, the thermal equilibration length of a vessel is a useful tool for examining which of the vessels are the equilibrating ones. The equilibration length is defined as the length after which the blood temperature in the vessel has approached the tissue temperature within $\mathrm{e}^{-1}$. For vessel types of capillaries, venules and arterioles, their equilibration length ranges are of the order of microns (Chen and Holmes 1980, Crezee and Lagendijk 1992). This implies that blood flowing in these small vessels will be completely equilibrated with the surrounding tissue. In this study we assume the blood temperature equals the tissue temperature in heated tissue so that the heat source/sink term $W_{\mathrm{b}} c_{\mathrm{b}}\left(T_{\mathrm{t}}-T_{\mathrm{b}}\right)$ will vanish. Based on this assumption, the volumetric averaging of the energy conservation principle can be achieved by combining and rearranging equation (1) multiplied by $(1-\phi)$ and equation (2) multiplied by $\phi$ with the porous structure regarded as a homogeneous medium to obtain

$$
\begin{aligned}
{\left[\phi \rho_{\mathrm{b}} c_{\mathrm{b}}+(1-\phi) \rho_{\mathrm{t}} c_{\mathrm{t}}\right] \frac{\partial T}{\partial t}+\phi \rho_{\mathrm{b}} c_{\mathrm{b}}\left[u \frac{\partial T}{\partial x}+v \frac{\partial T}{\partial y}+w \frac{\partial T}{\partial z}\right] } \\
=\left[\phi k_{\mathrm{b}}+(1-\phi) k_{\mathrm{t}}\right]\left(\frac{\partial^{2} T}{\partial x^{2}}+\frac{\partial^{2} T}{\partial y^{2}}+\frac{\partial^{2} T}{\partial z^{2}}\right)+(1-\phi) q_{\mathrm{t}}+\phi q_{\mathrm{b}}
\end{aligned}
$$

The porosity, $\phi$, is defined as the ratio of blood volume to the total (solid tissue + liquid blood) volume. During the computer simulation, the ultrasound power was uniformly deposited within the desired heating volume and the absorption of ultrasound power by the blood is 
assumed to be $10 \%, q_{\mathrm{b}}=0.1 q_{\mathrm{t}}$, of that for the tissue (Duck et al 1998). Hence, the total absorbed power density $q_{\mathrm{a}}$ is equal to $(1-0.9 \phi) q_{\mathrm{t}}$. We assumed that the initial and boundary temperatures of a $10 \times 10 \times 10 \mathrm{~mm}^{3}$ heating target cube and of its outside tissue volume were given as $T(x, y, z, 0)=37^{\circ} \mathrm{C}$, as shown in figure $1(\mathrm{~b})$.

\subsection{Dimensionless parameters}

In order to derive a compact representation, the following parameters are defined:

$$
\begin{aligned}
k_{\mathrm{eff}} & =\phi k_{\mathrm{b}}+(1-\phi) k_{\mathrm{t}} \quad \alpha^{*}=\frac{k_{\mathrm{eff}}}{\phi \rho_{\mathrm{b}} c_{\mathrm{b}}+(1-\phi) \rho_{\mathrm{t}} c_{\mathrm{t}}} \\
\alpha_{\mathrm{eff}} & =\frac{k_{\mathrm{eff}}}{\rho_{\mathrm{b}} c_{\mathrm{b}}} \quad q^{*}(x, y, z, t)=\phi q_{\mathrm{b}}(x, y, z, t)+(1-\phi) q_{\mathrm{t}}(x, y, z, t) .
\end{aligned}
$$

The dimensionless parameters have the following forms:

$$
X=\frac{x}{l} \quad Y=\frac{y}{l} \quad Z=\frac{z}{l} \quad T^{*}=\frac{T-T_{\mathrm{a}}}{T_{\mathrm{a}}} \quad \tau=\frac{\alpha^{*} t}{l^{2}}
$$

where $T_{\mathrm{a}}$ is the arterial blood temperature, which is set to $37^{\circ} \mathrm{C}$.

Substituting equations (4) and (5) into equation (3), equation (3) can be expressed in dimensionless form as

$\frac{\partial T^{*}}{\partial \tau}=\frac{\partial^{2} T^{*}}{\partial X^{2}}+\frac{\partial^{2} T^{*}}{\partial Y^{2}}+\frac{\partial^{2} T^{*}}{\partial Z^{2}}-2 c_{1} \frac{\partial T^{*}}{\partial X}-2 c_{2} \frac{\partial T^{*}}{\partial Y}-2 c_{3} \frac{\partial T^{*}}{\partial Z}+q_{1}(X, Y, Z, \tau)$

where

$c_{1}=\frac{u^{*} l}{2 \alpha_{\mathrm{eff}}} \quad c_{2}=\frac{v^{*} l}{2 \alpha_{\mathrm{eff}}} \quad c_{3}=\frac{w^{*} l}{2 \alpha_{\mathrm{eff}}} \quad u^{*}=\phi u \quad v^{*}=\phi v \quad w^{*}=\phi w$

$q_{1}(X, Y, Z, \tau)=\frac{q^{*}(X, Y, Z, \tau) l^{2}}{T_{\mathrm{a}} k_{\mathrm{eff}}}$.

By setting the product form as

$$
T^{*}(X, Y, Z, \tau)=\bar{T}(X, Y, Z, \tau) \exp \left(c_{1} X+c_{2} Y+c_{3} Z-c_{4} \tau\right)
$$

where

$$
c_{4}=c_{1}^{2}+c_{2}^{2}+c_{3}^{2}+\gamma
$$

and substituting it into equation (6), another simple form can be obtained as:

$$
\frac{\partial \bar{T}}{\partial \tau}=\frac{\partial^{2} \bar{T}}{\partial X^{2}}+\frac{\partial^{2} \bar{T}}{\partial Y^{2}}+\frac{\partial^{2} \bar{T}}{\partial Z^{2}}+q_{2}(X, Y, Z, \tau)
$$

where

$$
q_{2}(X, Y, Z, \tau)=q_{1}(X, Y, Z, \tau) \exp \left[-\left(c_{1} X+c_{2} Y+c_{3} Z-c_{4} \tau\right)\right] .
$$

Since the Green's function obtained for the differential equation is independent of the source term, the same Green's function can be used to calculate the temperature distribution for various sources with different spatial and temporal profiles. Therefore, once the Green's function is known, the temperature distribution in the medium is readily calculated. For an infinite medium $(-\infty<X<\infty,-\infty<Y<\infty,-\infty<Z<\infty)$ and for time $0<\tau$, the desired Green's function is not difficult to find (Özisik 1993) and is given by

$G\left(X, Y, Z, \tau \mid X_{\mathrm{p}}, Y_{\mathrm{p}}, Z_{\mathrm{p}}, \tau_{\mathrm{p}}\right)=\left[4 \pi\left(\tau-\tau_{\mathrm{p}}\right)\right]^{-\frac{3}{2}} \exp \left[-\frac{\left(X-X_{\mathrm{p}}\right)^{2}+\left(Y-Y_{\mathrm{p}}\right)^{2}+\left(Z-Z_{\mathrm{p}}\right)^{2}}{4\left(\tau-\tau_{\mathrm{p}}\right)}\right]$ 
where the positions and time with the subscript $\mathrm{p}$ refer to a heating power location. The Green's function $G\left(X, Y, Z, \tau \mid X_{\mathrm{p}}, Y_{\mathrm{p}}, Z_{\mathrm{p}}, \tau_{\mathrm{p}}\right)$ represents the dimensionless temperature $\bar{T}(X, Y, Z, \tau)$, at the location $(X, Y, Z)$, at time $\tau$, due to an instantaneous heat source, located at $\left(X_{\mathrm{p}}, Y_{\mathrm{p}}, Z_{\mathrm{p}}\right)$, releasing its energy spontaneously at time $\tau_{\mathrm{p}}$. The solution of equation (10) can be written in terms of the above Green's function, according to equation (11), as

$$
\begin{gathered}
\bar{T}\left(X, Y, Z, \tau \mid X_{\mathrm{p}}, Y_{\mathrm{p}}, Z_{\mathrm{p}}, \tau_{\mathrm{p}}\right)=\int_{\tau_{\mathrm{s}}}^{\tau_{\mathrm{f}}} \int_{X_{\mathrm{s}}}^{X_{\mathrm{f}}} \int_{Y_{\mathrm{s}}}^{Y_{\mathrm{f}}} \int_{Z_{\mathrm{s}}}^{Z_{\mathrm{f}}} G\left(X, Y, Z, \tau \mid X_{\mathrm{p}}, Y_{\mathrm{p}}, Z_{\mathrm{p}}, \tau_{\mathrm{p}}\right) \\
\times q_{2}\left(X_{\mathrm{p}}, Y_{\mathrm{p}}, Z_{\mathrm{p}}, \tau_{\mathrm{p}}\right) \mathrm{d} Z_{\mathrm{p}} \mathrm{d} Y_{\mathrm{p}} \mathrm{d} X_{\mathrm{p}} \mathrm{d} \tau_{\mathrm{p}}
\end{gathered}
$$

where the positions and time with the subscripts $\mathrm{s}$ and $\mathrm{f}$ refer to the start and finish of the computational domain, respectively. The approximating solution of equation (13) can be solved by the Gauss-Legendre integration method. Since Legendre polynomials are defined only in the interval $[-1,1]$, it is necessary to change the interval of integration for any arbitrary integral by making the substitution form

$$
\begin{array}{ll}
X_{\mathrm{p}}=\frac{\left(X_{\mathrm{f}}-X_{\mathrm{s}}\right) X_{\mathrm{p}}^{\prime}+X_{\mathrm{f}}+X_{\mathrm{s}}}{2} & Y_{\mathrm{p}}=\frac{\left(Y_{\mathrm{f}}-Y_{\mathrm{s}}\right) Y_{\mathrm{p}}^{\prime}+Y_{\mathrm{f}}+Y_{\mathrm{s}}}{2} \\
Z_{\mathrm{p}}=\frac{\left(Z_{\mathrm{f}}-Z_{\mathrm{s}}\right) Z_{\mathrm{p}}^{\prime}+Z_{\mathrm{f}}+Z_{\mathrm{s}}}{2} & \tau_{\mathrm{p}}=\frac{\left(\tau_{\mathrm{f}}-\tau_{\mathrm{s}}\right) \tau_{\mathrm{p}}^{\prime}+\tau_{\mathrm{f}}+\tau_{\mathrm{s}}}{2} .
\end{array}
$$

Applying the variable transformations that are expressed as equation (14) to equation (13), we can obtain another form:

$$
\begin{aligned}
\bar{T}\left(X, Y, Z, \tau \mid X_{\mathrm{p}}^{\prime}, Y_{\mathrm{p}}^{\prime}, Z_{\mathrm{p}}^{\prime}, \tau_{\mathrm{p}}^{\prime}\right)=\frac{\left(X_{\mathrm{f}}-X_{\mathrm{s}}\right)}{2} \frac{\left(Y_{\mathrm{f}}-Y_{\mathrm{s}}\right)}{2} \frac{\left(Z_{\mathrm{f}}-Z_{\mathrm{s}}\right)}{2} \frac{\left(\tau_{\mathrm{f}}-\tau_{\mathrm{s}}\right)}{2} \\
\quad \times \int_{-1}^{1} \int_{-1}^{1} \int_{-1}^{1} \int_{-1}^{1} G\left(X, Y, Z, \tau \mid X_{\mathrm{p}}^{\prime}, Y_{\mathrm{p}}^{\prime}, Z_{\mathrm{p}}^{\prime}, \tau_{\mathrm{p}}^{\prime}\right) \\
\times q_{2}\left(X_{\mathrm{p}}^{\prime}, Y_{\mathrm{p}}^{\prime}, Z_{\mathrm{p}}^{\prime}, \tau_{\mathrm{p}}^{\prime}\right) \mathrm{d} Z_{\mathrm{p}}^{\prime} \mathrm{d} Y_{\mathrm{p}}^{\prime} \mathrm{d} X_{\mathrm{p}}^{\prime} \mathrm{d} \tau_{\mathrm{p}}^{\prime} .
\end{aligned}
$$

Finally, it can be considered to define the transient and three-dimensional analytical solution form of equation (3) as

$$
\begin{aligned}
T(x, y, z, t)= & T_{\mathrm{a}}\left\{1+\frac{\left(X_{\mathrm{f}}-X_{\mathrm{s}}\right)\left(Y_{\mathrm{f}}-Y_{\mathrm{s}}\left(Z_{\mathrm{f}}-Z_{\mathrm{s}}\right)\left(\tau_{\mathrm{f}}-\tau_{\mathrm{s}}\right)\right.}{16}\right. \\
& \times \int_{-1}^{1} \int_{-1}^{1} \int_{-1}^{1} \int_{-1}^{1}\left[4 \pi\left(\tau-\tau_{\mathrm{p}}^{\prime}\right)\right]^{\frac{-3}{2}} q_{1}(X, Y, Z, \tau) \\
& \times \exp \left[c_{1}\left(X-X_{\mathrm{p}}^{\prime}\right)+c_{2}\left(Y-Y_{\mathrm{p}}^{\prime}\right)+c_{3}\left(Z-Z_{\mathrm{p}}^{\prime}\right)-c_{4}\left(\tau-\tau_{\mathrm{p}}^{\prime}\right)\right. \\
& -\frac{\left.\left.\left(X-X_{\mathrm{p}}^{\prime}\right)^{2}+\left(Y-Y_{\mathrm{p}}^{\prime}\right)^{2}+\left(Z-Z_{\mathrm{p}}^{\prime}\right)^{2}\right] \mathrm{~d} Z_{\mathrm{p}}^{\prime} \mathrm{d} Y_{\mathrm{p}}^{\prime} \mathrm{d} X_{\mathrm{p}}^{\prime} \mathrm{d} \tau_{\mathrm{p}}^{\prime}\right\}}{4\left(\tau-\tau_{\mathrm{p}}^{\prime}\right)} .
\end{aligned}
$$

An analytic formulation has been derived for the time-dependent and three-dimensional spatialdependent porous medium bioheat transfer equation. The exact solution can be used to predict the effect of blood flow on the transient temperature distribution of heated tissues without thermally significant vessels during thermal therapies. The approximate solution of equation (16) can be evaluated by the Gauss-quadrature integration scheme (Abramowitz and Stegun 1965). 

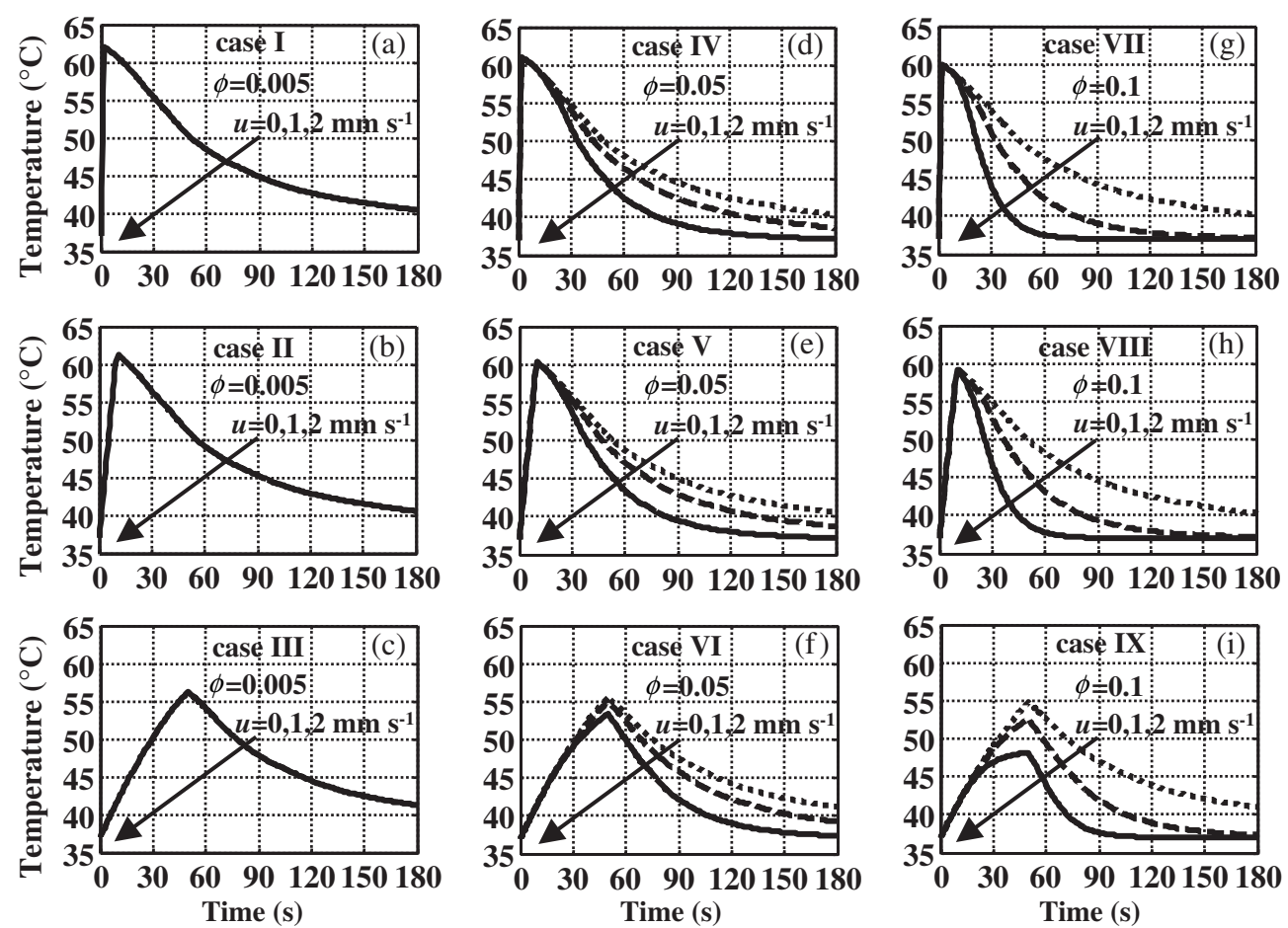

Figure 2. Effects of the heating schemes in table 2 on the transient profiles of the temperature at the central point within a heated cube with the nine heating schemes and for the three different blood averaged velocities $u=0,1$ and $2 \mathrm{~mm} \mathrm{~s}^{-1}$, were presented. The dotted, dashed and solid lines represented the blood averaged velocities $u=0,1$ and $2 \mathrm{~mm} \mathrm{~s}^{-1}$, respectively.

\subsection{Thermal dose calculations}

The thermal dose or equivalent minutes at $43{ }^{\circ} \mathrm{C}$ defined by Sapareto and Dewey (1984) can describe the extent of thermal damage or destruction of tissue, which is a function of the temperature elevation and time. The Sapareto-Dewey expression can be written as

$$
\mathrm{EM}_{43}(\text { in min })=\int R^{(T-43)} \mathrm{d} t
$$

where $R=2$ for $T \geqslant 43{ }^{\circ} \mathrm{C}$ and $R=4$ for $37{ }^{\circ} \mathrm{C}<T<43{ }^{\circ} \mathrm{C}, T$ is the temperature, $t$ is the time and $\mathrm{EM}_{43}$ is the thermal dose equivalence in minutes. The threshold values of thermal dose for necrosis are $\mathrm{EM}_{43}=25 \mathrm{~min}$ and $240 \mathrm{~min}$ for brain and muscle tissues, respectively (Sapareto and Dewey 1984, Damianou and Hynynen 1993). In this simulation study, a conservative value $\left(\mathrm{EM}_{43}=300 \mathrm{~min}\right)$ has been taken, which is similar to the analysis of Damianou and Hynynen (1993) for focused ultrasound surgery and Diederich and Burdette (1996) for prostate ultrasound thermal therapy.

\section{Results and discussion}

Figure 2 shows that the temperature at the centre of the heating target keeps increasing during the heating and then decays immediately if the heating power is turned off. Due to the assumption that the absorption of ultrasound power density by the blood is only $10 \%$ of that 

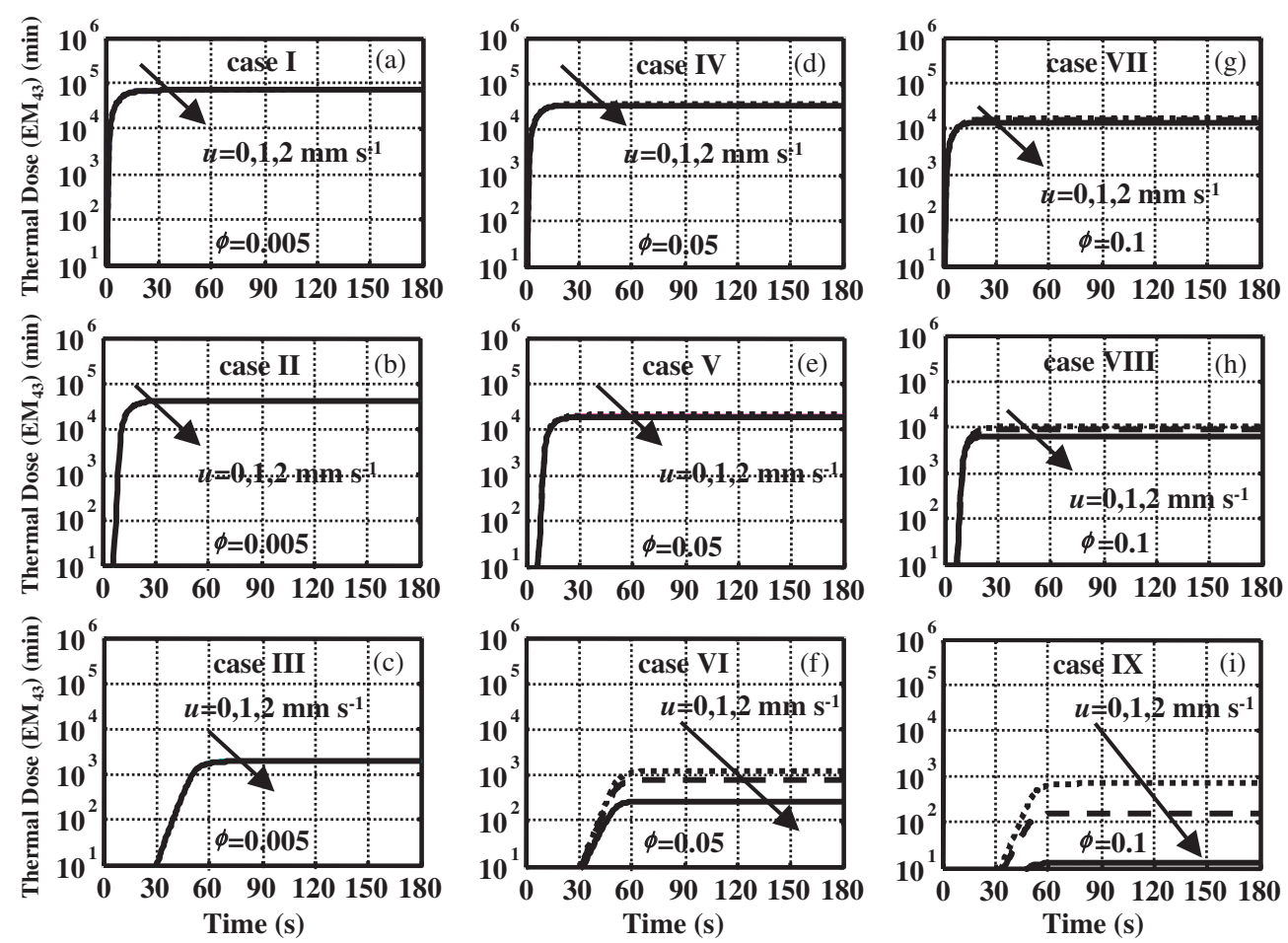

Figure 3. Effects of the heating schemes in table 2 on the transient profiles of the thermal dose equivalence $\left(\mathrm{EM}_{43}\right)$ corresponding to figure 2 are presented. The dotted, dashed and solid lines represent the blood averaged velocities $u=0,1$ and $2 \mathrm{~mm} \mathrm{~s}^{-1}$, respectively.

Table 2. Parameters of the different heating schemes used in simulation.

\begin{tabular}{|c|c|c|c|c|c|c|c|c|c|}
\hline Case & I & II & III & IV & V & VI & VII & VIII & IX \\
\hline Porosity & \multicolumn{3}{|c|}{$\phi=0.005$} & \multicolumn{3}{|c|}{$\phi=0.05$} & \multicolumn{3}{|c|}{$\phi=0.1$} \\
\hline $\begin{array}{l}\text { Absorbed power density for } \\
\text { soft tissue } q_{\mathrm{t}}\left(\mathrm{W} \mathrm{cm}^{-3}\right)\end{array}$ & 50 & 10 & 2 & 50 & 10 & 2 & 50 & 10 & 2 \\
\hline $\begin{array}{l}\text { Total absorbed power density } \\
q_{\mathrm{a}}=(1-0.9 \phi) q_{\mathrm{t}}\left(\mathrm{W} \mathrm{cm}^{-3}\right)\end{array}$ & 49.775 & 9.955 & 1.991 & 47.75 & 9.55 & 1.91 & 45.5 & 9.1 & 1.82 \\
\hline Heating duration (s) & 2 & 10 & 50 & 2 & 10 & 50 & 2 & 10 & 50 \\
\hline Total absorbed energy density $\left(\mathrm{J} \mathrm{cm}^{-3}\right)$ & \multicolumn{3}{|c|}{99.55} & \multicolumn{3}{|c|}{95.5} & \multicolumn{3}{|c|}{91} \\
\hline
\end{tabular}

by the tissue, a higher porosity leads to a lower total absorbed energy density which is also shown in table 2. Hence, the time-varying temperature distributions of higher porosity are lower than those of lower porosity when the heating duration and blood velocity maintain the same input. Under the same porosity and total absorbed power density, a more rapid heating results in a higher peak temperature. This implies that the peak temperature strongly depends upon the heating duration. For instance, the peak temperatures are about $62{ }^{\circ} \mathrm{C}$ in case I and $56{ }^{\circ} \mathrm{C}$ in case III shown in figures 2 (a) and (c). When the porosity is less than 0.005 , figures 2(a)-(c) and figures 3(a)-(c) reveal that the results of time-varying temperature distributions and thermal dose level are independent of the blood velocity. Except for the cases VI and IX, the peak temperatures shown in figure 2 and thermal dose levels shown in 

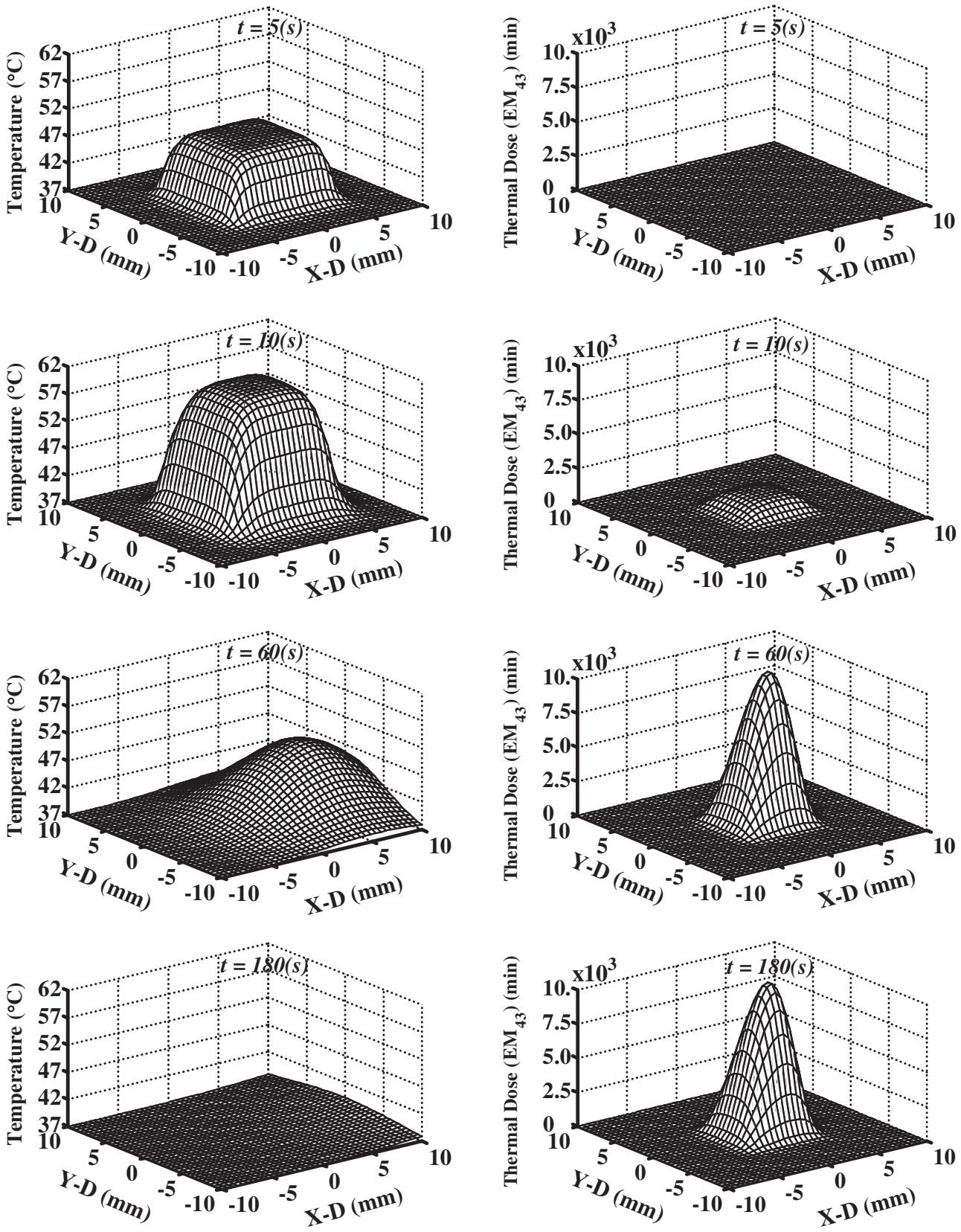

Figure 4. Temperature distributions and their corresponding thermal dose distributions on the $x-y$ plane with the heating case VIII and for the averaged blood velocity $u=1 \mathrm{~mm} \mathrm{~s}^{-1}$ along the $x$-axis direction at $t=5,10,60$ and $180 \mathrm{~s}$, respectively, are presented. Power was turned off at $10 \mathrm{~s}$. The porosity $\phi$ is 0.1 (Hilmas and Gillette 1974).

figure 3 are nearly independent of the blood velocity. In other words, the peak temperature and thermal dose level are significantly influenced by the blood velocity only if both longer heating duration and higher porosity exist. Billard et al (1990) also mentioned that the 


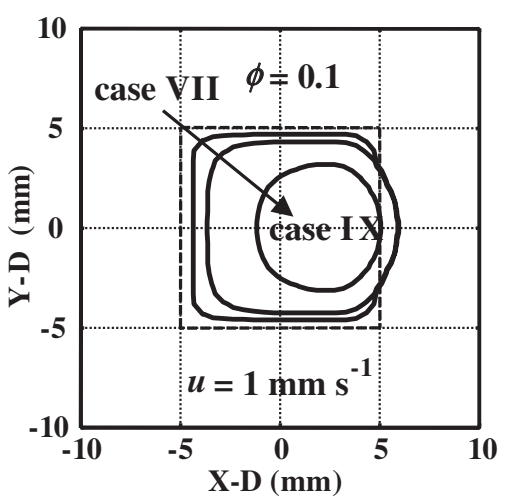

Figure 5. Effects of the different heating schemes on thermal dose equivalence $\mathrm{EM}_{43}=300$ contours at $t=180 \mathrm{~s}$ for averaged blood velocity $1 \mathrm{~mm} \mathrm{~s}^{-1}$. The dashed lines are the boundary of the heating cube.

influence of blood perfusion on thermal distributions is less sensitive for a short ultrasound pulse. In addition, figure 3 illustrates that the accumulation of $\mathrm{EM}_{43}$ reaches its plateau faster for a rapid heating duration, and the level of the plateau is proportional to the peak temperature.

Figure 4 demonstrates the transient behaviour of temperature distribution and thermal dose profile on the $x-y$ plane for case VIII. During the heating process, the elevation of temperature distribution is proportional to the heating time ranging from 0 to $10 \mathrm{~s}$. The peak temperature reaches about $59{ }^{\circ} \mathrm{C}$ when the power is off. Then, the plateau of temperature distribution shifts towards the downstream region of blood flow and decays with time. The elevation of thermal dose profile (i.e., $\mathrm{EM}_{43}$ ) mainly depends on the peak temperature. Consequently, the above results indicate that the temperature variation and thermal dose accumulation within the heating tissue depend on the heating duration, the porosity, the averaged blood velocity and the direction of blood flow during thermal therapy.

In order to further investigate the effect of the heating duration on the domain of thermal lesion $\left(\mathrm{EM}_{43}=300 \mathrm{~min}\right)$, the averaged blood velocity was set at $1 \mathrm{~mm} \mathrm{~s}^{-1}$. Figure 5 depicts that the contours of the $\mathrm{EM}_{43}=300$ min for cases VII and VIII almost coincide with each other downstream. This is due to the blood flow effect that transports the input thermal energy from upstream to downstream along the blood flow direction. Furthermore, the effective lesion domain for case IX is located on the right half portion of the heating target. Meanwhile, this area is obviously below $50 \%$ of the treatment target which we want to cure. Under the same porosity which induces the same amount of total absorbed energy density, the domain of thermal lesion that covers the desired therapeutic region for the shorter heating duration is larger than that for the longer heating duration. The effects with different heating schemes on thermal prediction have shown that a rapid heating scheme produces both higher peak temperature and larger thermal dose level. This phenomenon was also validated by the results from Torvi and Dale (1994) and Chen et al (1993). It is also found that the thermal dose level on the front portion of the treatment target cannot approach the therapeutic level, especially for a longer heating duration. Comparison of the heating schemes under the same porosity (i.e., the same total absorbed energy density) shows that a rapid heating scheme can produce a higher thermal dose level on the front portion of the heating target. Therefore, rapid heating can obtain more useful therapeutic results in terms of thermal dose level, and the blood flow 

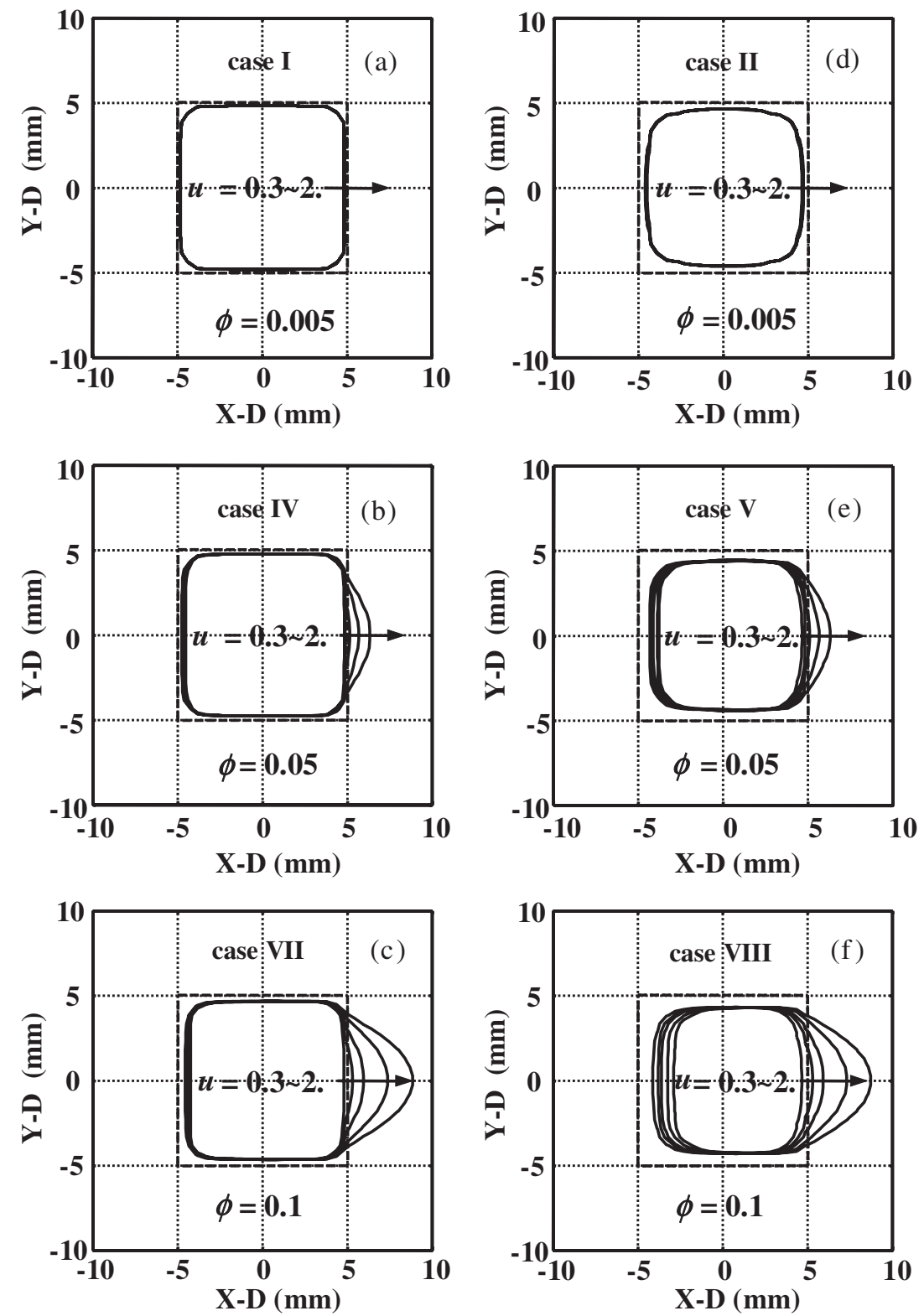

Figure 6. Effects of the porosity and averaged blood velocity on the thermal dose equivalence $\mathrm{EM}_{43}=300 \mathrm{~min}$ contours at $t=180 \mathrm{~s}$ with different porosity values of $0.005,0.05,0.1$, averaged blood velocities of $0.3,0.7,1.0,1.5,2 \mathrm{~mm} \mathrm{~s}^{-1}$, two heating patterns. Heating durations were $2 \mathrm{~s}$ for (a), (b) and (c), and $10 \mathrm{~s}$ for (d), (e) and (f). (a) and (d) with total absorbed energy density $99.55 \mathrm{~J} \mathrm{~cm}^{-3}$; (b) and (e) with total absorbed energy density $95.5 \mathrm{~J} \mathrm{~cm}^{-3}$ and (c) and (f) with the total absorbed energy density $91 \mathrm{~J} \mathrm{~cm}^{-3}$.

direction can drastically affect the domain and location of the thermal lesion during thermal therapy.

To study the effects of the porosity values ranging from 0.005 to 0.1 and the averaged blood flow velocities ranging from 0.3 to $2 \mathrm{~mm} \mathrm{~s}^{-1}$ on the thermal dose distributions, two 
groups of rapid heating schemes (cases I, IV and VII and cases II, V and VIII in table 2) are presented for comparison. When the porosity is 0.005 , figures $6(\mathrm{a})$ and (d) demonstrate that the contour of thermal lesion of $\mathrm{EM}_{43}=300 \mathrm{~min}$ is nearly independent of the magnitude of the blood velocity. When the averaged blood velocity is $0.3 \mathrm{~mm} \mathrm{~s}^{-1}$, figures 6 (a)-(c) show that the thermal dose equivalence contours of $\mathrm{EM}_{43}=300 \mathrm{~min}$ inside the heating target are almost identical with various inputs of porosity and heating duration. Although the domain of thermal lesion can effectively cover the wanted therapeutic region by using rapid heating, figures 6(b) and (c) show that thermal lesion on normal tissue will occur when the blood velocity and the porosity are increasing. Higher values of the porosity and the blood velocity in the rapid heating treatment can not only fully cover the therapeutic region, but also induce larger areas of overheating on normal tissue downstream of the flow direction. Similar results appear in figures 6(e) and (f).

\section{Conclusion}

By applying the porous medium model to describe the heat transfer in living tissue, an analytical solution can be obtained by means of a Green's function. The exact solution can be used to predict the effect of directional blood flow on the transient three-dimensional temperature distribution and thermal dose quantity of heated tissues during thermal therapy. The blood flow has the potential of transporting the deposition energy from the heating target to the normal tissue along the flow direction, especially with higher values of velocity and porosity. A higher porosity causes a lower total absorbed energy density. Under the same porosity, the lower peak temperature associated with its lower thermal dose level is acquired for the longer heating. Regarding rapid heating, there is no influence on temperature distribution and thermal dose profile when the averaged blood velocity is below $0.3 \mathrm{~mm} \mathrm{~s} \mathrm{~m}^{-1}$ or the porosity is below 0.005 . For higher porosity and a larger blood velocity, the domain of the thermal lesion may cover not only the wholly wanted therapeutic region but even go beyond to the normal tissue along the flow direction.

\section{Acknowledgments}

The work was supported in part by the National Science Council of Taiwan under contract no NSC 89-2213-E-036-014.

\section{References}

Abramowitz M and Stegun I A 1965 Handbook of Mathematical Functions with Formulas, Graphs, and Mathematical Tables (New York: Dover) pp 916-9

Baish J W 1994 Formulation of a statistical model of heat transfer in perfused tissue J. Biomech. Eng. 116 521-7

Baish J W, Netti P A and Jain R K 1997 Transmural coupling of fluid flow in microcirculatory network and interstitium in tumors Microvasc. Res. 53 128-41

Beacco C M, Mordon S R and Bruneaud J M 1994 Development and experimental in vivo validation of mathematical modeling of laser coagulation Lasers Surg. Med. 14 362-73

Billard B E, Hynynen K and Roemer R B 1990 Effects of physical parameters on high temperature ultrasound hyperthermia Ultrason. Med. Biol. 16 409-20

Chen L, Haar G, Hill C R, Dworkin M, Carnochan P, Young H and Bensted J P M 1993 Effect of blood perfusion on the ablation of liver parenchyma with high-intensity focused ultrasound Phys. Med. Biol. 38 1661-73

Chen M M and Holmes K R 1980 Microvascular contributions in tissue heat transfer Ann. NY Acad. Sci. 335 137-50

Crezee J and Lagendijk J J 1992 Temperature uniformity during hyperthermia: the impact of large vessels Phys. Med. Biol. 37 1321-37

Damianou C and Hynynen K 1993 Focal spacing and near-field heating during pulsed high temperature ultrasound therapy Ultrason. Med. Biol. $19777-87$ 
Damianou C A, Hynynen K and Fan X 1995 Evaluation of accuracy of a theoretical model for predicting the necrosed tissue volume during focused ultrasound surgery IEEE Trans. Ultrason. Ferroelectr. Freq. Control 42 182-7

Daum D R and Hynynen K 1999 A 256-element ultrasonic phased array system for the treatment of large volumes of deep seated tissue IEEE Trans. Ultrason. Ferroelectr. Freq. Control 46 1254-68

Diederich C J and Burdette E C 1996 Transurethral ultrasound array for prostate thermal therapy initial studies IEEE Trans. Ultrason. Ferroelectr. Freq. Control 43 1011-22

Duck F A, Baker A C and Starrit H C 1998 Ultrasound in Medicine (London: Institute of Physics Publishing) pp 57-88

Fan X and Hynynen K 1996 A study of various parameters of spherically curved phased arrays for noninvasive ultrasound surgery Phys. Med. Biol. 41 591-608

Folkman J 1986 How is blood vessel growth regulated in normal and neoplastic tissue? G H A Clowes memorial award lecture Can. Res. 46 467-73

Gao B, Langer S and Corry P M 1995 Application of the time-dependent Green's function and Fourier transforms to the solution of the bioheat equation Int. J. Hyperth. $11267-85$

Guyton A C 1986 The systemic circulation Textbook of Medical Physiology (Philadelphia, PA: Saunders) pp 218-20

Hilmas D E and Gillette E L 1974 Morphometric analyses of the microvascular of tumors during growth and after X-irradiation Cancer 33 103-10

Hirst D G 1989 Tumor Blood Flow Modification Therapeutic Benefit: Is this Approach Ready for Clinical Application? (London: Cancer Research Campaign) pp 14-7

Hirst D G, Denekamp J, Hirst V K, Joiner B, Peters C E, Prise V E, Sensky P L, Shaffi K M, Tozer G M and Ward A E M 1992 Tumor Vascular Physiology (London: Cancer Research Campaign) pp 40-6

Hobbie R K 1997 Intermediate Physics for Medicine and Biology 3rd edn (New York: Springer) p 19

Keller K H and Seiler L J 1971 An analysis of peripheral heat transfer in man J. Appl. Phys. 30 779-86

Kolios M C, Sherar M D and Hunt J W 1995 Large blood vessel cooling in heated tissues: a numerical study Phys. Med. Biol. 40 477-94

Kolios M C, Sherar M D and Hunt J W 1996 Blood flow cooling and ultrasonic lesion formation Med. Phys. 23 1287-98

Kolios M C, Worthington A E, Holdsworth D W, Sherar M D and Hunt J W 1999 An investigation of the flow dependence of temperature gradients near large vessels during steady state and transient tissue heating Phys. Med. Biol. 44 1479-97

Molls M and Vaupel P (ed) 1998 Blood Perfusion and Microenvironment of Human Tumors: Implications for Clinical Radiooncology (Berlin: Springer) pp 193-207

Nilsson G E 1996 Laser Doppler perfusion imaging 18th Ann. Int. Conf. of the IEEE Engineering in Medicine and Biology Society (Amsterdam) pp 196-7

Overgaard J, Gonzalez D G, Hulshof M C, Arcangeli G, Dahl O, Mella O and Bentzen S M 1996 Hyperthermia as an adjuvant to radiation therapy of recurrent or metastatic malignant melanoma. A multicentre randomized trial by the European Society for Hyperthermic Oncology Int. J. Hyperth. 12 3-20

Özisik M N 1993 Heat Conduction 2nd edn (New York: Wiley) pp 214-56

Pennes H H 1948 Analysis of tissue and arterial blood temperatures in the resting human forearm J. Appl. Phys. 1 93-122

Sapareto S A and Dewey W 1984 Thermal dose determination in cancer therapy Int. J. Radiat. Oncol. Biol. Phys. 10 787-800

Shubik P 1982 Vascularization of tumor: a review J. Can. Res. Clin. Oncol. 103 211-26

Thomsen S 1991 Pathologic analysis of photothermal and photomechanical effects of laser-tissue interactions Photochem. Photobiol. 53 825-35

Torvi D A and Dale J D 1994 A finite element model of skin subjected to a flash fire ASME J. Biomed. Eng. 116 $250-5$

Wan H, VanBaren P, Ebbini E S and Cain C A 1996 Ultrasound surgery: comparison of strategies using phased array systems IEEE Trans. Ultrason. Ferroelectr. Freq. Control 43 1085-98

Weinbaum S, Jiji L and Lemons D E 1984a Theory and experiment for the effect of vascular microstructure on surface tissue heat transfer: part I. Anatomical foundation and model conceptualization ASME J. Biomed. Eng. $106312-30$

Weinbaum S, Jiji L and Lemons D E 1984b Theory and experiment for the effect of vascular microstructure on surface tissue heat transfer: part II. Model formulation and solution ASME J. Biomed. Eng. 106 $331-41$

Wu X and Sherar 2002 Theoretical evaluation of moderately focused spherical transducers and multi-focus acoustic lens/transducer systems for ultrasound thermal therapy Phys. Med. Biol. 47 1603-21

Wulff W 1974 The energy conservation equation for living tissue IEEE Trans. Biomed. Eng. 21 494-5 